\title{
Reactogenicidad e inmunogenicidad tras la inoculación de vacuna frente a SARS-CoV-2 en personal de diálisis
}

\author{
Elena Guerrero-Rodríguez ${ }^{1}$, David Hernán-Gascueña², María Blanca Miranda-Serrano² ${ }^{2}$, Lola Arenas-Jiménez ${ }^{2}$, \\ Concepción Pereira-Feijoo ${ }^{3}$, Marta Sanjuan-Miguelsanz ${ }^{3}$
}

${ }^{1}$ Fundación Renal Íñigo Álvarez de Toledo. Centro Los Llanos 2. Getafe. Madrid. España

${ }^{2}$ Fundación Renal Íñigo Álvarez de Toledo. Madrid. España

${ }^{3}$ Fundación Renal Íñigo Álvarez de Toledo. Centros Orense. Orense. España

${ }^{4}$ Fundación Renal Íñigo Álvarez de Toledo. Centro Los Olmos. Segovia. España

Como citar este artículo:

Guerrero-Rodríguez E, Hernán-Gascueña D, Miranda-Serrano MB, Arenas-Jiménez L, Pereira-Feijoo C, Sanjuan-Miguelsanz M. Reactogenicidad e inmunogenicidad tras la inoculación de vacuna frente a SARS-CoV-2 en personal de diálisis. Enferm Nefrol. 2021 Jul-Sep;24(3):262-70

\section{Resumen}

Introducción: Ser personal sanitario en activo supone un factor de riesgo elevado para contraer la infección por SARS-CoV-2. La realización de programas de vacunación es la mejor herramienta disponible para enfrentar este problema. En España, los profesionales sanitarios han sido vacunados de manera prioritaria.

Objetivo: Evaluar, a través del recuento de anticuerpos en sangre, la inmunogenicidad de las vacunas frente a SARS-CoV-2 en personal de diálisis y los factores relacionados con ella.

Material y Método: Estudio observacional descriptivo de corte trasversal, multicéntrico. Se realizó una determinación serológica del recuento de anticuerpos totales anti-proteína " $\mathrm{S}$ " en las 12 semanas posteriores a la vacunación. La recogida de datos se realizó a través de un cuestionario ad-hoc, online, anónimo y voluntario.

Resultados: 167 participantes, todos vacunados con vacunas ARN. EL 15\% había pasado previamente la infección. El 96,4\% han desarrollado anticuerpos (media de $15.776 \pm 13.640 \mathrm{AU} / \mathrm{ml}$ ); aquellos profesionales que han pasado previamente la infección tienen un número pro-

\section{Correspondencia:}

Elena Guerrero Rodríguez

E-mail: eguerrero@friat.es medio de anticuerpos significativamente mayor que los que no $(23.532$ vs $14.381 \mathrm{AU} / \mathrm{ml})(p=0,05)$. La presencia de síntomas como cansancio, cefalea, fiebre y mialgias se asocia, también, a una reactividad significativamente mayor $(p=0,004)$. No existe relación significativa entre la reactividad y sexo, edad, índice de masa corporal (IMC) o lugar de trabajo.

\section{Conclusiones:}

La inmunogenicidad provocada por las vacunas ARN administradas a personal de diálisis ha sido casi total. Padecer efectos adversos post vacunación, como cansancio, cefaleas, fiebre, náuseas y dolores, y haber pasado previamente la infección se asocia con mayor reactividad, manifestada con el desarrollo de mayor número de anticuerpos.

PALABRAS CLAVE: coronavirus; SARS-CoV-2; vacuna; personal sanitario; hemodiálisis.

\section{Reactogenicity and immunogenicity after inoculation of SARS-CoV-2 vaccine in dialysis care workers}

\section{Abstract}

Introduction: Being an active healthcare worker is a high-risk factor for contracting the SARS-CoV-2 infection. The implementation of vaccination programs 
is the best option available to solve this problem. In Spain, healthcare professionals have been vaccinated as a matter of priority.

Objective: To assess, through blood antibody counts, the immunogenicity of SARS-CoV2 vaccines in dialysis personnel and related factors.

Material and Method: Observational, cross-sectional and multicentric descriptive study.

Results: 167 participants, all vaccinated with RNA vaccines. $15 \%$ have previously passed the infection. $96.4 \%$ have developed antibodies (mean of 15,776 $+/-13,640$ ). Those professionals who have previously passed the infection have a significantly higher average number of antibodies than those who have not $(23,532$ vs 14,381$)(p=0.05)$. The presence of symptoms such as fatigue, headache, fever, and myalgia is also related with a significantly greater reactivity $(p=0.004)$. There is no significant relationship between reactivity and sex, age, BMI, or workplace.

Conclusions: Immunogenicity caused by RNA vaccines administered to dialysis personnel has been almost complete. Suffering post-vaccination adverse effects such as fatigue, headaches, fever, nausea, pain, and having previously passed the infection is related to a greater reactivity, manifested by the development of an increased number of antibodies.

KEYWORDS: coronavirus; SARS-CoV-2; vaccine; health care worker; hemodialysis.

\section{Introducción}

Desde que se conocieran los primeros casos de infección por SARS-CoV-2, la investigación farmacológica ha centrado sus esfuerzos en desarrollar vacunas seguras y eficaces frente a esta enfermedad, lo que ha hecho posible que a principios de 2021 comenzara la vacunación en nuestro país. El desarrollo de las vacunas se ha realizado en un tiempo récord y para ello ha sido necesario acortar los tiempos programados para los ensayos con las mismas. La realización de programas de vacunación masivos es, hasta ahora, la mejor herramienta disponible para poder alcanzar una nueva situación que permita una óptima convivencia con la enfermedad ${ }^{1}$.

Hasta el 8 de marzo de 2021, se habían autorizado en España (previamente por la Agencia Europea de Medi- camentos) tres vacunas frente a la COVID-19. Comirnaty, de Pfizer/BioNTech, Moderna y AstraZeneca. Las tres vacunas han demostrado niveles adecuados de eficacia y seguridad en ensayos clínicos controlados. Las dos primeras tienen como componente principal al ARNm que codifica para la producción de la proteína S de SARS-CoV- $2^{2}$.

Ser personal sanitario en activo supone, por sí mismo, pertenecer a los grupos catalogados como de riesgo ante la infección por SARS-CoV-2 ${ }^{1}$. Según el estudio "Riesgo de infección por SARS-CoV-2 en trabajadores sanitarios", realizado a trabajadores sanitarios en activo en Italia, estos presentan un elevado porcentaje de contagio frente a SARS-CoV-2, probando, además, que existe una diferencia significativa de contagio entre aquellos profesionales que están en contacto directo con pacientes positivos respecto a aquellos que no lo están ${ }^{3}$. Según la Sociedad Española de Nefrología (SEN) el $20 \%$ de los nefrólogos en España han sufrido ya la COVID-194. Por este motivo el personal de los servicios de Nefrología ha sido considerado e incluido en el Grupo 2 de vacunación según la Estrategia de vacunación frente a COVID-19 en España ${ }^{5}$. Este grupo incluye al personal que tiene mayor riesgo de exposición por llevar a cabo actividades de atención directa a pacientes, así como por tener mayor probabilidad de tener contacto con una persona con infección por SARS-CoV-2².

El personal sanitario puede contagiarse tanto en el ámbito sanitario, como en su ámbito personal. El contagio del personal sanitario por esta enfermedad supone una gran preocupación para las autoridades sanitarias, ya que tiene un doble efecto sobre el Sistema Nacional de Salud: la escasez de personal sanitario activo para cubrir la alta demanda de ingresos hospitalarios y la posibilidad de contagio tanto al resto de personal sanitario, como a pacientes y a su propio entorno cercano ${ }^{6}$.

En un estudio realizado a personal sanitario en Israel, se analizó la efectividad de la vacuna de Pfizer en los primeros 14 días o entre los días 15 a 28 tras recibir la primera dosis, comparando los vacunados frente a los no vacunados. La tasa de reducción de la infección por SARS-CoV-2 fue del $30 \%$ en el primer periodo y del $75 \%$ en el segundo ${ }^{7}$.

Otro estudio realizado en Reino Unido, que analizaba la efectividad y cobertura de la vacuna Pfizer en sanitarios de hospitales públicos, mostró una efectividad en la prevención de infección, tanto sintomática como asintomática, del $72 \%$ a los 21 días de la primera dosis y del $86 \%$ pasados 7 días tras la segunda dosis ${ }^{8}$. 
Ambos estudios refuerzan la efectividad y la importancia de la vacunación, teniendo en cuenta el papel indiscutible que el personal sanitario está teniendo durante la pandemia ${ }^{1}$.

Respecto a los posibles acontecimientos adversos que se pueden producir tras la vacunación frente a SARSCoV-2, el Sistema Español de Farmacovigilancia de Medicamentos de Uso Humano (SEFV-H) realiza una valoración permanente de los mismos que han sido notificados en España. Estos acontecimientos adversos se registran en la base de datos FEDRA ${ }^{\circledR}$ con el objetivo de identificar de manera temprana nuevas reacciones adversas para analizarlas en profundidad ${ }^{2}$.

Basándonos en los datos publicados en la Estrategia de Vacunación frente a COVID-19 en España actualizada en febrero de $2021^{5}$, los efectos adversos más comunes tras la vacunación son, según la vacuna administrada:

- Pfizer: el dolor en el lugar de inyección ( $>80 \%)$, fatiga o sensación de cansancio $(>60 \%)$, cefalea $(>50 \%)$, mialgias y escalofríos ( $>30 \%)$, artralgias $(>20 \%)$, fiebre e inflamación en el lugar de inyección (>10\%).

- Moderna: el dolor en el lugar de inyección (92\%), fatiga $(70 \%)$, cefalea $(64,7 \%)$, mialgias $(61,5 \%)$, artralgias $(46,4 \%)$, escalofríos $(45,4 \%)$, náuseas/ vómitos $(23 \%)$, fiebre $(15,5 \%)$, inflamación en el lugar de inyección (14,7\%).

- AstraZeneca: sensibilidad en el lugar de inyección $(63,7 \%)$, dolor en el lugar de inyección $(54,2 \%)$, fatiga $(53,1 \%)$, cefalea $(52,6 \%)$, mialgias $(44 \%)$, artralgias $(26,4 \%)$, escalofríos $(31,9 \%)$, nauseas $(21,9 \%)$, fiebre $(7,9 \%)$.

Con las vacunas de Pfizer y Moderna las reacciones son más frecuentes tras la segunda dosis, lo contrario que ocurre con la vacuna de AstraZeneca que presenta reacciones más leves y menos frecuentes tras la segunda dosis. En las tres la frecuencia e intensidad de las mismas disminuye con la edad?.

Destacar que actualmente se desconoce, con seguridad, la totalidad de los efectos secundarios que pueden provocar. Por lo que, son necesarios estudios como el que nos ocupa, que permitan llegar a conocer la totalidad de su alcance ${ }^{10}$

Por tanto, el objetivo principal del presente estudio es evaluar, a través del recuento de anticuerpos en sangre, la inmunogenicidad de las vacunas frente a SARS-CoV-2 en personal de diálisis y los factores relacionados con ella, con los objetivos secundarios siguientes:

1. Analizar las reacciones adversas presentadas tras la vacunación de la última dosis y su relación con distintas variables.

2. Analizar el efecto de la infección por SARS-CoV-2 sobre la tasa de anticuerpos desarrollados post vacunación.

\section{Material y Método}

Se ha llevado a cabo un estudio observacional, descriptivo, de corte transversal, multicéntrico, realizado a los profesionales sanitarios, grupo de apoyo al paciente y personal de limpieza pertenecientes a las unidades hospitalarias y extrahospitalarias gestionadas por la Fundación Renal Íñigo Álvarez de Toledo (FRIAT) durante el primer semestre de 2021.

Los sujetos participantes han sido seleccionados entre el personal de los 18 centros gestionados por la Fundación Renal Íñigo Álvarez de Toledo, siempre que cumplieran los criterios de inclusión establecidos. En el momento en el que se recopilaron los datos para el estudio, la Fundación Renal contaba con un total de 282 profesionales en activo, que desempeñaban su trabajo en primera línea. A través de una base de datos facilitada por el departamento de Recursos Humanos, se accedió al correo electrónico corporativo de todos los trabajadores. A todos ellos se les envió un correo donde se les informaba del propósito del estudio, los criterios de inclusión del mismo y se les facilitó una breve explicación de cómo cumplimentar el cuestionario que recibirían posteriormente.

Del total de profesionales en activo, 247 (el 87,6\%) habían sido vacunado, al menos, con la primera dosis frente a SARS-CoV-2, en el momento de iniciar el estudio. Todos los participantes fueron vacunados con vacunas de ARNm (Pfizer o Moderna). En la tabla 1, está representada la distribución del total de profesionales y las frecuencias absolutas y relativas de vacunación.

\section{Criterios de inclusión:}

Todo el personal sanitario de primera línea vacunado frente a SARS-CoV-2, que trabajara en cualquiera de los centros extrahospitalarios o unidades hospitalarias de diálisis gestionados por la Fundación Renal Íñigo Álvarez de Toledo durante el primer semestre de 2021. 
Tabla 1. Porcentaje de profesionales vacunados según categoría profesional.

\begin{tabular}{|lc|c|c|}
\hline & $\begin{array}{c}\text { Personal } \\
\text { total }\end{array}$ & $\begin{array}{c}\text { Personal } \\
\text { vacunado }\end{array}$ & $\begin{array}{c}\text { Porcentaje } \\
\text { vacunación }\end{array}$ \\
\hline Enfermero & 143 & 126 & 88,11 \\
\hline $\begin{array}{l}\text { Técnico en cuidados } \\
\text { auxiliares de enfermería }\end{array}$ & 86 & 76 & 88,37 \\
\hline Médico/Nefrólogo & 23 & 18 & 78,26 \\
\hline Grupo de apoyo al paciente & 7 & 7 & 100 \\
\hline Limpieza & 23 & 20 & 86,96 \\
\hline Total & 282 & 247 & $\mathbf{8 7 , 5 9}$ \\
\hline
\end{tabular}

\section{Criterios de exclusión:}

Se ha excluido a todo el personal que no había sido vacunado cuando se llevó a cabo la recogida de datos, que no quiso realizarse la determinación serológica o que no cumplimentó el cuestionario online.

Las variables analizadas fueron: la edad de los participantes, el sexo (varón o mujer), altura, peso, índice de masa corporal (IMC). Además, se ha analizado el tipo de unidad donde trabaja (centro de diálisis extrahospitalario o unidad hospitalaria de nefrología). También se ha recogido la fecha de vacunación, si había pasado previamente la infección por SARS-CoV-2 (en caso de haberla pasado, la fecha en la que fue diagnosticado) y el recuento de anticuerpos que tenían según la serología realizada en sangre.

Respecto a los efectos adversos presentados post vacunación, se ha valorado la presencia de la siguiente sintomatología: fiebre, cansancio, cefalea, mareo, diarrea, náuseas, dolor muscular o mialgia o dolor en la zona de punción. Además, se ha habilitado una respuesta abierta donde se puede indicar cualquier otro síntoma que no haya sido recogido anteriormente.

Para detectar la reactogenicidad de las vacunas se realizó un cuestionario, emitido en función de la fecha de vacunación. Se trataba de un cuestionario adhoc, anónimo y voluntario, realizado de manera online. Dicho cuestionario estaba compuesto por 9 preguntas con múltiples respuestas en la que se incluía: fecha de administración de la vacuna, efectos secundarios presentados post vacunación, recuento de anticuerpos y diagnóstico previo positivo confirmado de COVID-19 (incluyendo la fecha de diagnóstico), así como variables demográficas y datos antropométricos. Los síntomas post vacunación que recoge el cuestionario incluyen
Ios valorados por el Sistema Español de Farmacovigilancia de la Agencia Española del Medicamento, incluidos en la base de datos FEDRA ${ }^{\circledR}$, respecto a la vacunación frente a SARS-CoV-2.

Para evaluar la inmunogenicidad de las vacunas se realizó una determinación serológica voluntaria y anónima a cada participante. Las muestras de sangre obtenidas se analizaron mediante un inmunoanálisis quimioluminiscente de micropartículas (CMIA), utilizado para la determinación cualitativa y cuantitativa de anticuerpos IgG frente al SARS-CoV-2 en suero y plasma humanos en el ARCHITECT i System de Abbott. A través de ellas, se identificaba el recuento de anticuerpos totales anti proteína "S" durante las 12 primeras semanas posteriores a la vacunación. Los valores de referencia de dicha determinación fijan el punto de corte en $50 \mathrm{AU} / \mathrm{ml}$, siendo un resultado inferior a este negativo y un resultado mayor o igual a 50 $\mathrm{AU} / \mathrm{ml}$ positivo ${ }^{11}$. Todas las muestras fueron extraídas a cada profesional en su centro de referencia, posteriormente se centrifugaron y conservaron en neveras entre $2^{\circ} \mathrm{C}$ y $8^{\circ} \mathrm{C}$. A continuación, todas las muestras fueron transportadas por una empresa de transporte especializado y analizadas en un mismo laboratorio, que se encuentra acreditado para la realización de análisis clínicos y pertenece a un hospital de tercer nivel.

Los participantes podían acceder al resultado de la serología a través de la plataforma del laboratorio. Para ello tenían que darse de alta en la misma, dónde obtenían una clave que le daba acceso al resultado serológico. Posteriormente debían cumplimentar el dato obtenido en el cuestionario.

Los datos obtenidos se volcaron en una base de datos Excel Microsoft Office Profesional Plus 2019 donde se recogieron las diferentes variables a estudiar. Posteriormente fueron procesados con el programa $\mathrm{R}$ versión 4.0.4.

Se realizó un análisis descriptivo para definir las características de la población estudiada. La muestra fue descrita mediante la media y la desviación estándar para todas las variables cuantitativas y una distribución de frecuencias para las variables cualitativas. Se utilizó la t de Student para comparar grupos en las variables continuas y la prueba de chi-cuadrado para las variables categóricas. Las pruebas estadísticas fueron de dos colas y se asumió significación estadística para un valor de $p<0,05$. 


\section{Aspectos éticos y legales}

Este estudio se realiza de acuerdo con los principios éticos basados en la Declaración de Helsinki, y que son coherentes con la guía de Buena Práctica Clínica (BPC) de la Conferencia Internacional del Armonización (ICH), y la legislación vigente a nivel estatal y de cada Comunidad Autónoma. Todos los participantes dieron su consentimiento informado para participar en este estudio.

Los datos recogidos estarán protegidos por la ley actual de protección de datos (Ley Orgánica 3/2018, de 5 de diciembre, de Protección de Datos Personales y garantía de los derechos digitales) según marca la legislación vigente. El estudio garantiza que la información que se maneja no contenga datos de carácter personal.

\section{Resultados}

Fueron finalmente incluidos en el estudio 167 profesionales, correspondiente al $67,6 \%$ del total de profesionales vacunados. De ellos $130(77,84 \%)$ trabajaban en un centro de diálisis extrahospitalario, 35 (20,9\%) lo hacían en una unidad de nefrología hospitalaria y 2 (1,2\%) lo hacían en ambas. El 79,6\% (133) eran mujeres y el $20,4 \%$ (34) hombres. La edad media de los mismos fue de $41,13 \pm 11,96$ años y su índice de masa corporal $24,58 \pm 4,25 \mathrm{~kg} / \mathrm{m}^{2}$.

La media de días transcurridos desde que los profesionales recibieron la última dosis de vacunación hasta que se realizó el recuento de anticuerpos, fue $36 \pm 17$ días (rango: 1-89 días). El 96,4\% de los profesionales vacunados habían desarrollado anticuerpos. El número máximo de anticuerpos desarrollados fue 80.000 y el mínimo 0, situándose la media del recuento en 15.776 $\pm 13.640 \mathrm{AU} / \mathrm{ml}$ de anticuerpos. No existía asociación estadísticamente significativa entre el recuento de anticuerpos y la edad de los participantes, el sexo, el IMC, su lugar de trabajo o en el número de días que habían transcurrido entre la última dosis de vacunación y la extracción de la muestra (ver tabla 2).

El $15 \%$ de los profesionales que participaron en el estudio había pasado previamente la infección por SARS-CoV-2. Cabe destacar, que existe una asociación

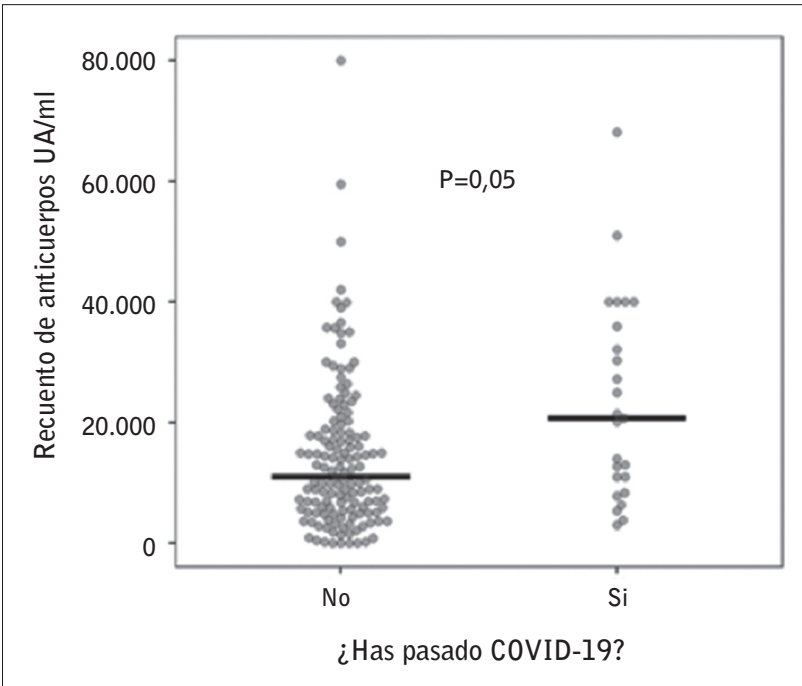

Figura 1. Recuento de anticuerpos según haya presentado o no Infección previa por COVID-19

estadísticamente significativa entre el hecho de haber pasado previamente la infección y la tasa de anticuerpos detectados, siendo mayor en las personas que habían pasado previamente la enfermedad $(23.532 \pm 16.595$ vs $14.381 \pm 12586 \mathrm{AU} / \mathrm{ml})(p=0,05)$ comparado con los que no la habían sufrido (ver figura 1 ).

Respecto a los efectos adversos que presentaron los participantes en el estudio, 143 profesionales tuvieron efectos adversos frente a 21 que no tuvieron dichos efectos. Al comparar el título de anticuerpos entre los profesionales con efectos adverso frente a los profesionales que no los presentaron las diferencias fueron 


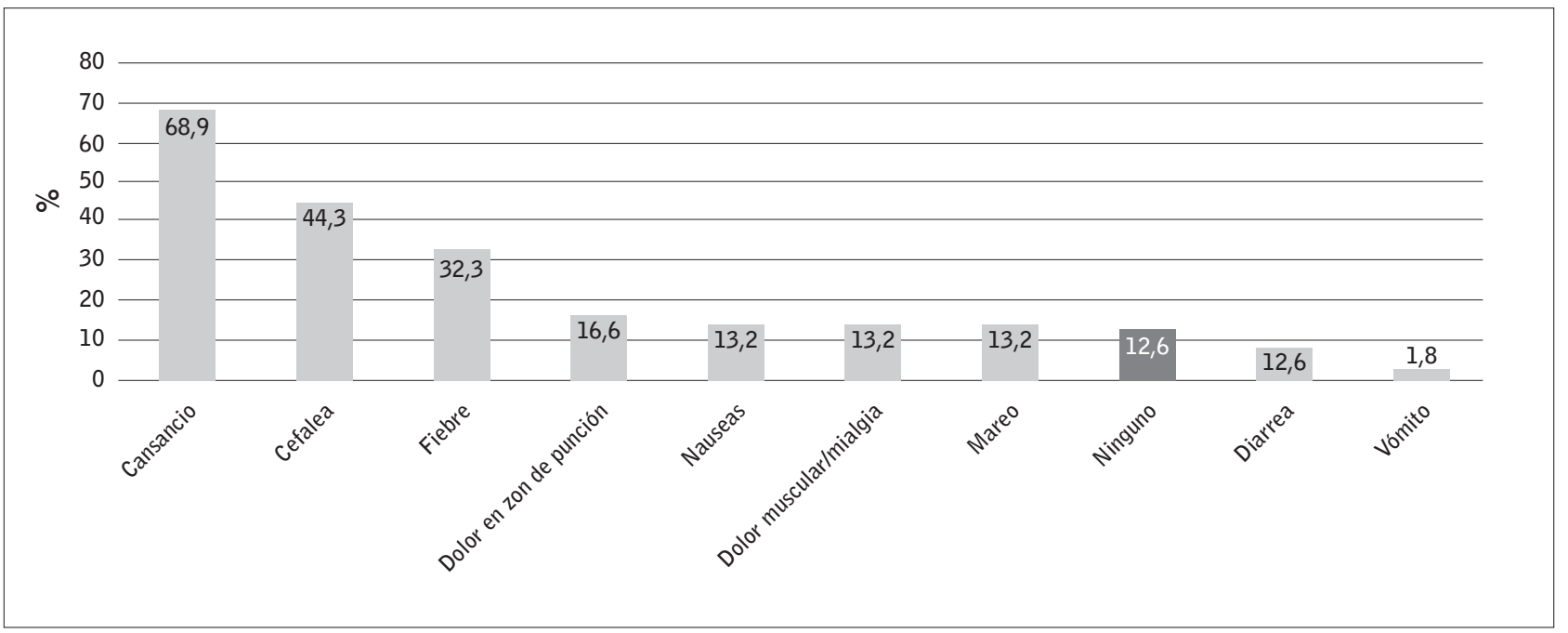

Figura 2. Porcentaje de efectos adversos manifestados.

estadísticamente significativas $(16.779 \pm 13.990 \mathrm{AU} /$ $\mathrm{ml}$ Vs $8.948 \pm 6.010 \mathrm{AU} / \mathrm{ml}, \mathrm{p}=0,004)$.

En la figura 2 se pueden observar los efectos adversos reportados por los trabajadores, según orden decreciente. Un $12,6 \%$ de los participantes refirió no haber tenido ningún efecto adverso tras la vacunación (ver figura 2).

La presencia de efectos adversos tales como el cansancio $(p=0,019)$, la cefalea $(p=0,021)$, la fiebre $(p=0,001)$, las náuseas $(p=0,018)$ y el dolor muscular/ mialgia $(p=0,0001)$, se asociaron significativamente con una mayor inmunogenicidad a la vacuna.

\section{Discusión}

Según nuestro estudio, el $15 \%$ de los profesionales que desempeñan su trabajo en unidades de diálisis ha sufrido la infección por SARS-CoV-2, lo que supone el doble de la tasa de contagio en la población general en España $(8 \%)^{12}$. Parece que queda demostrado que, al igual que indican otros estudios ${ }^{1,13}$, el hecho de ser profesional que desarrolla su trabajo en primera línea supone, por sí mismo, un factor de riesgo elevado a la hora de contraer la infección por SARS-CoV-2. Además, una reciente macroencuesta realizada por el Sindicato de Enfermería (SATSE) entre el personal de enfermería, indica que el $37 \%$ de este colectivo asegura haber sufrido síntomas propios de la enfermedad, porcentaje que contrasta con el del $10 \%$ que refiere la población española según el estudio nacio- nal de seroprevalencia realizado por el Ministerio de Sanidad ${ }^{14}$.

El contagio de los profesionales sanitarios provoca un doble efecto sobre el Sistema Nacional de Salud. Por un lado, es evidente la escasez que existe actualmente de personal sanitario en activo que pueda hacer frente a la elevada tasa de hospitalización provocada por la pandemia. Además, los profesionales sanitarios se enfrentan a un múltiple riesgo al contagiarse, el riesgo de contagio al resto de personal sanitario, a su propio entorno y a los pacientes que atienden. Es por ello que, desde la Sociedad Española de Nefrología, recomiendan la vacunación del personal sanitario que trata los pacientes con tratamiento renal sustitutivo, debido a la vulnerabilidad de los mismos ${ }^{4}$.

Actualmente, la vacunación masiva de la población se presenta como la mejor herramienta disponible para poder recuperar la normalidad que vivíamos previa a la llegada de la pandemia ${ }^{15}$. En este punto los profesionales sanitarios también juegan un papel importante, ya que ellos son el ejemplo en el que gran parte de la población se escuda a la hora de tomar decisiones acerca de la vacunación.

Respecto a la efectividad de la vacuna de ARNm, como hemos podido comprobar con nuestro estudio el desarrollo de anticuerpos ha sido casi total, con un $96,4 \%$, similar al de otros estudios realizados a personal de diálisis ( $100 \%$ en una muestra de 16 profesionales sanitarios) ${ }^{16}$. El número máximo de anticuerpos desarrollados por los participantes en el es- 
tudio ha sido $80.000 \mathrm{AU} / \mathrm{ml}$ (valor máximo que proporciona el recuento de anticuerpos según el sistema de quimioluminiscencia del ARCHITECT i System), situándose la media del recuento en $15.776 \mathrm{AU} / \mathrm{ml}$. Otros estudios realizados también a profesionales sanitario, evidencian la fuerte respuesta frente a vacunas de ARN, pero como el método de inmunoanálisis utilizado ha sido diferente (ChemiLuminescent ImmunoAssay- CLIA y chemiluminescent assay ADVIA Centaur ${ }^{\circledR}$ XP/XPT COV2G) ${ }^{16}$, no es posible comparar a nivel cuantitativo los valores con los obtenidos con los datos de nuestra muestra. Por otra parte, según indica un informe elaborado por el Comité científico sobre el COVID-19 del Colegio Oficial de Médicos de Madrid (ICOMEM), se desconoce cuál es el nivel necesario de anticuerpos que se ha de tener para estar protegido frente a la infección. Es más, refieren que no es necesario comprobar la respuesta inmunitaria mediante la medición de anticuerpos, ya que dicha respuesta puede variar de unos individuos a otros y no se pueden correlacionar, por tanto, los títulos individuales con el grado de protección frente a la enfermedad ${ }^{17}$. Además, se desconoce hasta el momento, cuál es la duración de la respuesta inmunitaria. Aunque se cree que la protección de la vacuna puede ser semejante a la ofrecida tras pasar la enfermedad de manera natural, el seguimiento de las personas que han sido incluidas en los ensayos clínicos realizados hasta el momento es muy corto.

Independientemente de los anticuerpos desarrollados y de la duración de los mismos, podemos afirmar que, al igual que ya adelantaban los estudios realizados en Israel $^{7,13}$ (país pionero en la vacunación frente a SARSCoV-2), las vacunas son eficaces. El uso de las misma y la aplicación de las medidas higiénico sanitarias recomendadas por las diferentes instituciones (distancia social, confinamientos, uso de mascarillas, lavado de manos...) han propiciado una reducción significativa en el número de contagios, muertes y hospitalizaciones tanto en personas de avanzada edad, como en personal sanitario y en la población general ${ }^{17-19}$.

La desconfianza en la seguridad de la vacuna y el riesgo de sufrir efectos adversos son dos de los motivos principales que apuntan los ciudadanos para rechazar la vacunación ${ }^{11}$. Aunque es cierto que, como cualquier vacuna, las vacunas frente a SARS-CoV-2 pueden provocar infrecuentes efectos adversos graves tras su inoculación, estos no debieran justificar la retirada del mercado de estas vacunas o la negativa a la administración de las mismas.
Los efectos secundarios recogidos en nuestro estudio, coinciden ampliamente con los notificados por la Agencia Española de Medicamentos y Productos Sanitarios. Según el último informe de farmacovigilancia de la vacuna ${ }^{19}$, publicado en mayo de 2021, los acontecimientos más frecuentemente notificados siguen siendo los trastornos generales (fiebre, dolor en la zona de vacunación), del sistema nervioso (cefalea, mareos) y del sistema músculo-esquelético (mialgia y artralgia). Hasta el 25 de abril la tasa de notificaciones de efectos adversos de las vacunas era de 121 notificaciones por cada 100.000 dosis administradas. De ellos un $18 \%$ se consideraron graves, entendiéndose grave todo efecto adverso que requiera o prolongue la hospitalización, dé lugar a una discapacidad significativa o persistente, ponga en peligro la vida, así como cualquier otra condición que se considere médicamente significativa.

En nuestro estudio parece probada la relación entre el hecho de haber pasado la infección previamente o el desarrollo de determinados síntomas post vacunación y una mayor reacción inmunológica. Esta relación también es avalada por el estudio Israelí realizado a principios de año "Impact of age gender ethnicity and prior disease status on immunogenicity following", realizado a personal sanitario, cuya muestra es superior a la de nuestro estudio (514 participantes). En este estudio, además, se encontraron diferencias significativas respecto a la edad de los participantes, siendo menos reactivos aquellos profesionales que cuentan con mayor edad ${ }^{20}$, lo que puede relacionarse también con menor gravedad en los efectos adversos desarrollados en las personas mayores ${ }^{9}$. Es cierto que los participantes de estos estudios no han sido seleccionados de manera aleatoria, por lo que los resultados no pueden ser extrapolados a la población general. Pero, estos resultados pueden servir como punto de partida de futuras investigaciones, que pueden ayudar a conocer la respuesta inmunológica de las vacunas sin la necesidad de realizar determinaciones serológicas a toda la población.

Añadir que, al igual que con otros estudios de corte transversal, nuestro estudio muestra la situación en un momento puntual y dados los continuos cambios referentes a todo lo que tiene que ver con la pandemia y la vacunación, los datos obtenidos pueden variar en un corto espacio de tiempo. Podría ser interesante realizar otro corte en meses posteriores para valorar la evolución respecto al recuento de anticuerpos a la vez que continuamos registrando las incidencias de la enfermedad y la situación epidemiológica. Además, contamos con la limitación de que todos los datos del estudio provienen del cuestionario que han de cumplimentar los participantes y hemos de- 
tectado valores en blanco y datos erróneos que han sido excluídos.

En cualquier caso, cabe destacar que la dedicación que los profesionales sanitarios han tenido durante la pandemia, en la que han atendido y proporcionado cuidados directos a personas infectadas con COVID-19, ha sido indudable. Al inicio de la pandemia no se disponía del conocimiento necesario de la enfermedad ni de las medidas de protección adecuadas para evitar el contagio por la misma. EI esfuerzo, extraordinario y de gran responsabilidad, llevado a cabo por estos profesionales ha tenido, tristemente, consecuencias para su salud física y psicológica.

Tras analizar los datos obtenidos en el estudio, podemos concluir que la inmunogenicidad provocada por las vacunas ARNm administradas a personal de diálisis ha sido casi total, por lo que la vacunación en este colectivo ha sido efectiva.

Los efectos adversos desarrollados post vacunación han sido mayoritariamente leves-moderados. Padecer efectos adversos post vacunación tales como cansancio, cefaleas, fiebre, náuseas y dolores, se ha asociado con el desarrollo de mayor número de anticuerpos en los profesionales analizados.

Existe también una relación significativa entre el hecho de haber pasado la infección previamente y la reacción inmunológica, ya que todos aquellos profesionales que habían estado previamente infectados por SARS-CoV-2 han tenido una mayor tasa de respuesta inmunológica.

Recepción: 02-07-21

Aceptación: 10-08-21

Publicación: 30-09-21

\section{Bibliografía}

1. Di Gennaro F, Murri R, Segala FV, Cerruti L, Abdulle A, Saracino A, et al. Attitudes towards Anti-SARSCoV2 Vaccination among Healthcare Workers: Results from a National Survey in Italy. Viruses (consultado 21 Feb 2021) Disponible en: https://doi. org/10.3390/v1303037113, (2021).

2. Grupo de Trabajo Técnico de Vacunación COVID-19, de la ponencia de programa y registro de vacunaciones. Estrategia de vacunación frente a COVID-19 en España. Actualización 5. (consultado 14 May 2021) Disponible en: https://www.mscbs.gob.es/profesionales/saludPublica/prevPromocion/vacunaciones/ covid19/docs/COVID-19_Actualizacion5_EstrategiaVacunacion.pdf (2021).

3. The Lancet. Risk for SARS-CoV-2 Infection in Healthcare Workers, Turin, Italy. The Lancet vol. 395 1011 (2020). (consultado 7 Mar 2021) Disponible en: https://www.ncbi.nlm.nih.gov/pmc/articles/ PMC7774556/.

4. Sánchez-Álvarez E, Quiroga B, de Sequera P. Posicionamiento de la Sociedad Española de Nefrología ante la vacunación frente al SARS-CoV-2. Nefrología (consultado 4 Feb 2021) Disponible en: https://www.senefro.org/contents/webstructure/noticias/Position_statement_Vacuna_COVID_\%20-\%20Copy\%201.pdf.

5. Estrategia de vacunación frente a COVID-19 en España. Consejo Interterritorial. Sistema Nacional de Salud. Abril 2021 [consultado 28 Abr 2021]. Disponible en: https://www.mscbs.gob.es/profesionales/ saludPublica/prevPromocion/vacunaciones/covid19/ docs/COVID-19_Actualizacion7_EstrategiaVacunacion.pdf.(2021).

6. Baker TL, Greiner JV, Maxwell-Schmidt E, Lamothe $\mathrm{PH}$, Vesonder M. Guidelines for Frontline Health Care Staff Safety for COVID-19. Journal of Primary Care and Community Health vol. 11 (2020) (consultado $3 \mathrm{Abr}$ 2021) Disponible en: https:// www.ncbi.nlm.nih.gov/pmc/articles/PMC7377597/ pdf/10.1177_2150132720938046.pdf.

7. Amit S, Regev-Yochay G, Afek A, Kreiss Y, Leshem E. Early rate reductions of SARS-CoV-2 infection and COVID-19 in BNT162b2 vaccine recipients. Lancet 2021[consultado 15 Mar 2021];6;397(10277):87577 Disponible en: https://www.thelancet.com/journals/lancet/article/PIIS0140-6736(21)00448-7/ fulltext.

8. Hall VJ, Foulkes S, Saei A, Andres N, Oguti B, Charlett $A$. Effectiveness of BNT162b2 mRNA vaccine against infection and COVID-19 vaccibe coverge in healthcare workers in England. Lancet Feb 2021 [En prensa]. Disponible en: https://papers.ssrn.com/ sol3/papers.cfm?abstract_id=3790399. 
9. Soiza RL, Scicluna C, Thomson EC. Efficacy and safety of COVID-19 vaccines in older people. Age and Ageing. 2021 Feb 26;50(2):279-83.

10. Klimek L, Bergmann KC, Brehler R, Pfützner W, Zuberbier T, Hartmann $K$, et al. Practical handling of allergic reactions to COVID-19 vaccines: A position paper from German and Austrian Allergy Societies AeDA, DGAKI, GPA and ÖGAI. Allergo J Int, 2021; 19:1-17. [En prensa].

11. Abbott. AdviseDx SARS-CoV-2 IgG II. [consultado 6 Mar 2021]. Disponible en: https://www.fda.gov/ media/146371/download.

12. Actualización $n^{\circ}$ 405. Enfermedad por el coronavirus (COVID-19). Ministero de Sanidad. Gobierno de España. Jun 2021 [consultado 26 may 2021]. Disponible en: https://www.mscbs.gob.es/profesionales/saludPublica/ccayes/alertasActual/nCov/documentos/ Actualizacion_405_COVID-19.pdf.

13. Mallapaty S. Vaccines are curbing COVID: Data from Israel show drop in infections. Nature. 2021 Feb;590(7845):197.

14. Estudio ENE-COVID: Cuarta Ronda. Estudio nacional de sero-epidemiología de la infección por SARS-CoV-2 en España. Instituto de Salud Carlos III, Ministerio de sanidad y Ministerio de ciencia e innovación. Dic 2020 [consultado 2 Mar 2021]. Disponible en: https://www.mscbs.gob.es/gabinetePrensa/notaPrensa/pdf/15.12151220163348113. pdf (2020).
15. Aschwanden C. Five reasons why herd immunity for COVID is probably impossible. Nature. 2021 Mar;591(7851):520-2.

16. Jahn M, Korth J, Dorsch O, Anastasiou OE, Roge-Hädicke Humoral response to SARS-CoV-2-vaccination with BNT162b2 (pfizer-biontech) in patients on hemodialysis. Vaccines (Basel). 2021 Apr 8;9(4):360.

17. Monográfico vacunación COVID-19. La realidad tras los ensayos clínicos. Comité Científico del ICOMEM sobre la COVID-19, 2021 [consultado 24 May 2021]. Disponible en: https://www.icomem.es/adjuntos/adjunto_3092.1618927955.pdf.

18. Estrategia de detección precoz, vigilancia y control de COVID-19. Instituto Carlos III. Ministerio de Sanidad. Gobierno de España. 2021 [consultado 2 Jun 2021]. Disponible en: https://www.mscbs.gob. es/profesionales/saludPublica/ccayes/alertasActual/ nCov/documentos/COVID19_Estrategia_vigilancia_y_control_e_indicadores.pdf.

19. Vacunas COVID-19. Informe de farmacovigilancia Número 5. Agencia Española de Medicamentos y Productos Sanitarios. 2021 [consultado 27 May 2021]. Disponible en: https://www.aemps.gob.es/ IaAEM PS/docs/informe-farmacovigilancia-mayo-2021.pdf?x16990.

20. Jabal KA, Ben-Amram H, Beiruti K, Sussan C, Zarka $\mathrm{S}$, et al. Impact of age gender ethnicity and prior disease status on immunogenicity following. MedRxiv. Enero 2021 [En prensa].

Este artículo se distribuye bajo una Licencia Creative Commons Atribución-NoComercial 4.0 Internacional. https://creativecommons.org/licenses/by-nc/4.0/ 